\title{
Glycemic Control of Stroke Patients and Their Outcomes in a Comprehensive Stroke Center at a Tertiary Care Hospital: A Retrospective Cohort
}

\author{
Mohammad Amir Hossain, Barbara Wyczesany, Dwyer Jackie, Swapnil Patel, Khushboo Agarwal, \\ Rabail Soomro, Brian Yung, Arda Akoluk, Stephen Martino, Jennifer Cheng*, Raquel Ong, \\ Arif Asif
}

Department of Medicine, Jersey Shore University Medical Center, Hackensack Meridian Health, Neptune, USA

Email address:

Jennifers.cheng@hackensackmeridian.org (J. Cheng)

*Corresponding author

\section{To cite this article:}

Mohammad Amir Hossain, Barbara Wyczesany, Dwyer Jackie, Swapnil Patel, Khushboo Agarwal, Rabail Soomro, Brian Yung, Arda Akoluk, Stephen Martino, Jennifer Cheng, Raquel Ong, Arif Asif. Glycemic Control of Stroke Patients and Their Outcomes in a Comprehensive Stroke Center at a Tertiary Care Hospital: A Retrospective Cohort. American Journal of Internal Medicine. Vol. 8, No. 3, 2020, pp. 133-137. doi: 10.11648/j.ajim.20200803.17

Received: April 17, 2020; Accepted: May 9, 2020; Published: May 28, 2020

\begin{abstract}
Background: Diabetes mellitus is one of the major modifiable risk factors for having a stroke and those who present with a stroke are found to have hyperglycemia. The association between hyperglycemia and stroke has been found in an increasing number of studies. Evidence indicates that persistent in-hospital hyperglycemia during the first 24 hours after acute ischemic stroke (AIS) is associated with worse outcomes than normoglycemia. We conducted a retrospective chart review to see the glycemic control of stroke patients and their hospital outcome over a 3-month period as part of a preevaluation assessment to develop a hyperglycemia protocol in the stroke unit. Methods: Institutional review board approval was obtained for this study. A total of 142 patients with acute stroke admitted in the Comprehensive Stroke Center at Jersey Shore University Medical Center over a 3-month period were included in this study. Demographic characteristics, comorbidities, glycemic patterns at admission and throughout the hospital stay, types of stroke, length of stay, NIH (National Institutes of Health) stroke scale on admission and discharge, discharge disposition and outcome were reviewed retrospectively. Statistical analysis was performed by SPSS and a nova with tukey as post-hoc analysis. For the graph, we used prism and for the regression analysis, stat disk was used. Results: Average age of the patients was 73 years, with male predominance (54\%). Average BMI of the patients was 28.2. Most of the patients had an ischemic stroke (73\%). Major comorbidities were hypertension (88\%), dyslipidemia (66\%), and diabetes (36\%). About 8 of $143(6 \%)$ patients were newly diagnosed with diabetes. Average HbalC was 6.5. For a detailed statistical analysis, we divided patients into three groups by their blood glucose levels ranging from $80-140 \mathrm{mg} / \mathrm{dl}$ in group 1, 140-180 mg/dl in group 2, and more than $180 \mathrm{mg} / \mathrm{dl}$ in group 3. Then, we compared the length of stay (LOS), NIH scale admission/discharge, types of stroke and discharge disposition within the three groups. This study showed that the NIH stroke scale on admission in group 3 (blood glucose $>180 \mathrm{mg} / \mathrm{dl}$ ) was higher compared to group 1 (blood glucose $80-140 \mathrm{mg} / \mathrm{dl}$ ), with a $\mathrm{p}$ value of $<0.01$. Length of stay was more in group 3 compared to group 1 and 2, with a $\mathrm{P}$ value $<0.08$. Conclusion: Although this study limited by smaller patient groups, but it concludes that higher blood glucose level associated with higher NIH stroke scale at admission and during discharge and have increased length of stay which is consistent with other similar studies
\end{abstract}

Keywords: Diabetes, Stroke, Hyperglycemia, Hypoglycemia 


\section{Introduction}

The incidence of diabetes mellitus is rapidly growing, and diabetes complications can increase the risk for many health conditions, including stroke. Diabetes and stroke represent a tremendous human and economic burden, and therefore, should be high on the agenda for health research in $21^{\text {st }}$ century [1]. Diabetes and strokes have emerged as an important public health priority.

Data from AHA 2013-2016, suggest that 26 million or $9.8 \%$ of American adults had diagnosed diabetes mellitus; 91.8 million $(37.6 \%)$ have prediabetes, and 9.4 million (3.7\%) have undiagnosed diabetes [2]. Diabetes is a wellrecognized independent but modifiable risk factor for stroke, both ischemic and hemorrhagic [3]. The association between hyperglycemia and stroke has been found in an increasing number of studies. Adults with diabetes have a 1.8 to 6 -fold increased risk for stroke compared with nondiabetic adults [4]. Prediabetes and newly diagnosed diabetes are common in patients with stroke, without known diabetes prior to the event [5]. The deleterious effects of diabetes on the vasculature are well recognized through various mechanisms, including damage to the layer of blood vessels, inflammation, and an enhanced thrombotic environment [6].

The early recognition of hyperglycemia in stroke patients is important, as multiple complications are preventable through proper management of patients with acute stroke [7]. Stroke is the fifth leading cause of death and the leading cause of serious disability in the U.S.A [8]. A stroke is defined by focal neurological symptoms that occur when blood flow to an area of brain is cut off, by a clot or rupture [3]. In the U.S.A, the incidence of stroke due to ischemia is about $87 \%$ and hemorrhage $13 \%$ [9]. Diabetes mellitus is one of the major modifiable risk factors for having a stroke and those who present with a stroke are found to have hyperglycemia. As hyperglycemia is affiliated with worse patient outcomes, proper management of blood glucose levels after acute stroke is challenging, but imperative [4]. The American Stroke Association (ASA) and the American Heart Association (AHA) have established guidelines that have led to recommendations in the process of stroke care, as evidenced by the development of clinical recommendations for glucose control in acute stroke patients $[10,11]$. Achievement of the recommended ASA/AHA clinical targets for the management of patients with acute stroke, such as maintaining the serum glucose concentration within the range of 140 to $180 \mathrm{mg} / \mathrm{dL}(7.8-10 \mathrm{mmol} / \mathrm{L})$ in a hospitalized stroke patient, has led to better patient outcomes.[10] Evidence indicates that persistent in - hospital hyperglycemia during the first 24 hours after acute ischemic stroke (AIS) is associated with worse outcomes than normoglycemia and thus, it is reasonable to treat hyperglycemia in patient with AIS. Hypoglycemia (blood glucose $<60 \mathrm{mg} / \mathrm{dl}$ ) should also be treated in patients with AIS $[10,11]$. We aim to do a retrospective chart review to see the glycemic control of stroke patients admitted in the stroke unit over a 3-month period and their subsequent hospital outcome. We are planning to develop a hyperglycemia management protocol in our Comprehensive Stroke Center as there is no current procedure guidelines in place; with this new protocol, we plan to educate the stroke nurses and supporting staff to aspire for better patient outcomes.

\section{Methods and Statistical Analysis}

\subsection{Methods}

Institutional review board approval was obtained for this study. A total of 142 patients with acute stroke admitted in the comprehensive stroke care center at Jersey Shore University Medical Center over a 3-month period were included in this study. Demographic characteristics, comorbidities, glycemic patterns at admission and throughout the hospital stay, types of stroke, length of stay, NIH (National Institutes of Health) stroke scale on admission and discharge, discharge disposition, and outcome were reviewed retrospectively from hospital record. Blood glucose levels were measured by the morning comprehensive metabolic panel (CMP) and glucometer readings, then documented as a range and average blood glucose on a daily basis. Depending on the blood glucose levels patients were divided into 3 groups. Group 1 included patients with BS of 80-140 $n=86$; Group 2 included patients with BS 140-180 $\mathrm{n}=31$; and Group 3 included patients with BS of 180 or more $n=25$. HbA1C was used according to the American Diabetic Association (ADA) recommendations to categorize the patients into those without diabetes $(<5.7 \%)$, those with prediabetes $(5.7 \%$ to $6.4 \%)$, and those with diabetes $(>6.5 \%)[8,12]$. Hypertension was defined as systolic blood pressure greater than or equal to $130 \mathrm{mmHg}$ and diastolic blood pressure greater than or equal to $90 \mathrm{mmHg}$ and /or current antihypertensive treatments. BMI was defined as underweight $(<18.5)$, normal weight (18.5-24.9), overweight (25.0-29.9), and obese ( $>30)$ [13]. Mean values were calculated for continuous variables and absolute, and relative frequencies were calculated for discrete variables.

\subsection{Statistical Analysis}

Statistical analysis was performed by SPSS (Statistical Package for the Social Sciences, Chicago, Illinois, USA). One- way-Anova test with Tukey-b as post-hoc tests were used for comparison between groups. For the graph, we used GraphPad Prism (GraphPad Software, San Diego California, USA) and for the regression analysis, we calculated Pearson Correlation coefficient and generated graphs using Statdisk (www.statdisk.org<http://www.statdisk.org>, Triola 2016). Data is presented as mean \pm SD and P values; $p$ values of $<$ 0.05 were accepted as statistically significant [14].

\section{Results}

A total 142 patients were admitted for cerebrovascular accident in the stroke unit at JSUMC over a 3- month period. 
The average age of these patients was 73 years of age, with average body mass (BMI) index of 28.2. Most predominant race was white $(79 \%)$. Of these 142 patients, 52 patients (36.4\%) had a diagnosis of diabetes mellitus prior to admission, and 8 patients were newly diagnosed on this admission. Other predominant comorbidities were hypertension $(86.7 \%)$, hyperlipidemia $(66.4 \%)$, coronary artery disease (35\%), history of arrhythmia (29\%) and history of previous cerebrovascular accident (28\%). Demographic characteristics are shown in Table 1.

$105(74 \%)$ patients were diagnosed with ischemic stroke and $37(26 \%)$ patients had hemorrhagic stroke. Out of 105 ischemic stroke patients, $37(35 \%)$ patients had interventions performed (17 patients received TPA and 20 received neurovascular/neurosurgical intervention). According to average blood glucose level, patients were categorized into 3 different groups. 61\% (87/143) of patients belonged to group 1 (average blood glucose level between $80-140 \mathrm{mg} / \mathrm{dl}), 22 \%(31 / 143)$ of patients in group 2 (average blood glucose level between 141-180mg/dl), and $17 \%$ of patients were in group 3 (average blood glucose level $>180 \mathrm{mg} / \mathrm{dl}$ ). See Table 2. Average admission NIH Stroke Scale was 8.9 and average discharge NIH Stroke Scale was 7.4. Average admission NIH Stroke Scale was higher in patients with higher average blood glucose levels. (Group 1 versus group2, $\mathrm{P}=0.01$ and group 1 versus group 3 , $\mathrm{p}=0.003)$. Average discharge change in NIH Stroke Scale was not statistically significant (Group 1 versus group 2, $\mathrm{P}=.0017$ and group 1 versus group $3 \mathrm{P}=0.008$ ), however the changes are seen in Graph 1. Length of Stay increased from 4.46 days in patients with blood glucose $<100$, compared to 10 days in patients with glucose $>180$. Nearly $44 \%$ patients of patients who had an average hospital glucose less than 120 were discharged to home, compared to $22.6 \%$ with an average glucose between 120-180, and 4\% with an average glucose more than 180 .

When considering BMI (body mass index), the average length of stay was 1.16 days higher in the obese patient (BMI>30) when compared to non-obese patients $(\mathrm{BMI}<30)$. Patients with BMI $>30$, (Table 3), had a higher average admission and discharge NIH scale (9.76 and 8.59 respectively). These differences were not statistically significant most likely due to our sample size. In this study, 9 patients had an incidence of hypoglycemia by finger stick during their hospital course.
Table 1. Demographic characteristics of the patient $(n=142)$.

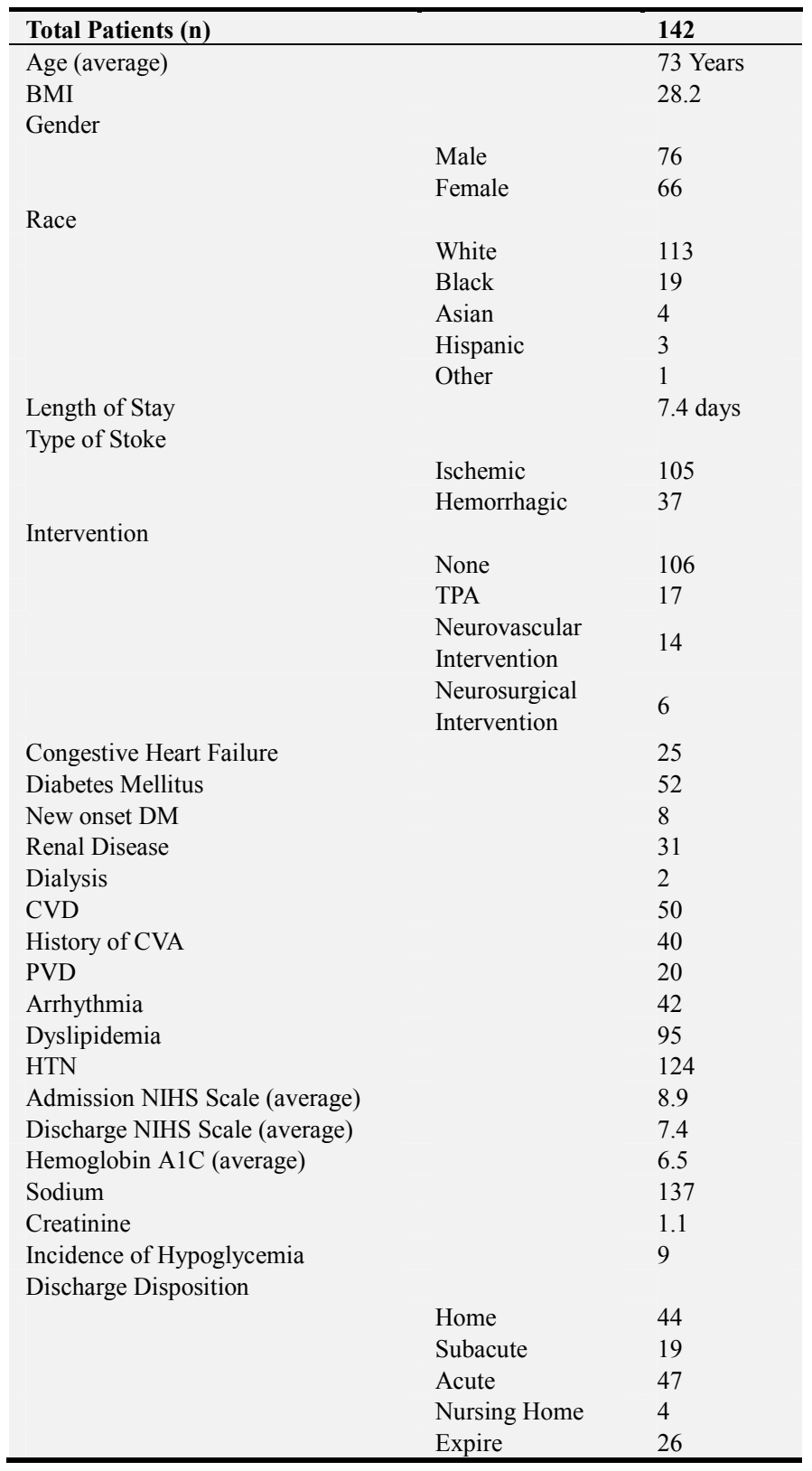

Table 2. Blood glucose level and outcome of the patient $(n=142)$.

\begin{tabular}{|c|c|c|c|}
\hline Average blood glucose level (mg/dl) & Group $1<100$ - $139(n=84)$ & Group 2140-180 $(n=31)$ & Group 3>180 $(n=25)$ \\
\hline Average Admission NIHSS & 6.21 & 11.9 & 14.64 \\
\hline Average Discharge NIHSS & 4.43 & 9.71 & 14.64 \\
\hline Average Change NIHSS & 1.77 & 2.19 & 0 \\
\hline Length of Stay & 6.28 days & 8.39 days & 10 \\
\hline \multicolumn{4}{|l|}{ Discharge Disposition } \\
\hline Home & 32 & 8 & 4 \\
\hline Subacute rehab & 12 & 5 & 2 \\
\hline Nursing Home & 2 & 0 & 2 \\
\hline Deceased & 7 & 11 & 10 \\
\hline
\end{tabular}


Table 3. Outcomes of patients based on BMI $(n=142)$.

\begin{tabular}{lll}
\hline BMI & $<\mathbf{3 0}(\mathbf{n = 8 8})$ & $>\mathbf{3 0}(\mathbf{n}=\mathbf{5 4 )}$ \\
\hline Length of Stay & 6.94 days & 8.1 days \\
Average NIH on admission & 8.44 & 9.76 \\
Average NIH on Discharge & 6.64 & 8.59 \\
Change in NIH & 1.8 & 1.2 \\
Blood Sugar Average & 132 & 147.7 \\
Discharge Disposition & & 17 \\
Home & 27 & 9 \\
Sub-Acute & 10 & 15 \\
Acute Rehab & 32 & 1 \\
Nursing Home & 3 & 12 \\
Deceased & 16 & \\
\hline
\end{tabular}

Table 1

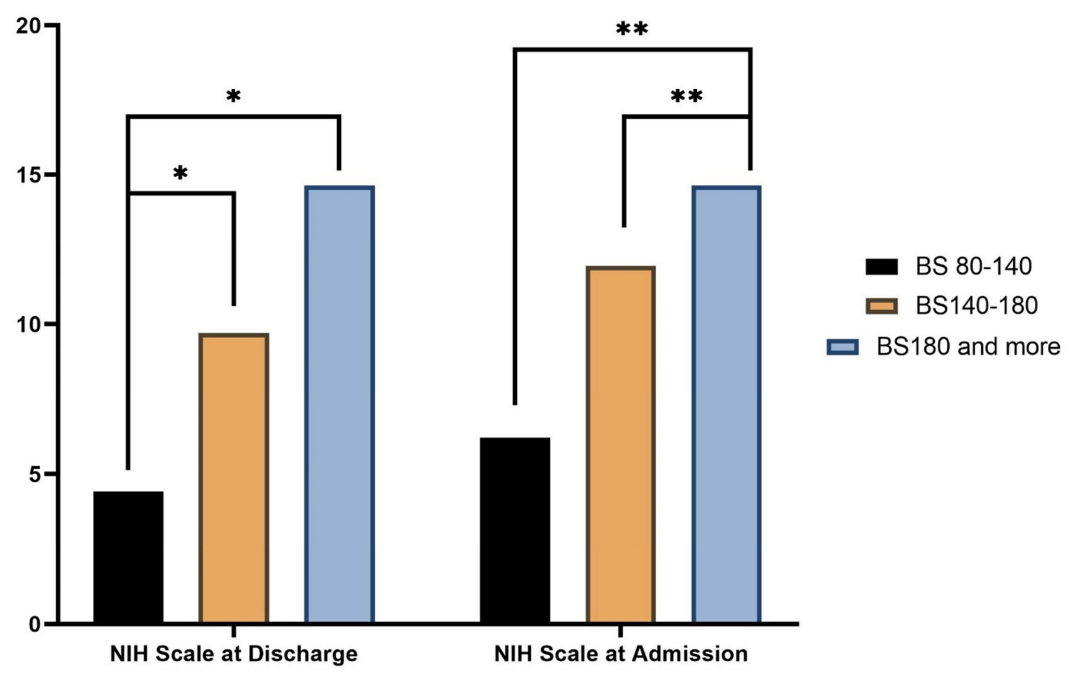

Figure 1. Blood glucose level and NIH scale ( $n=142)$.

\section{Discussion}

Glycemic control has a major impact on the outcomes of patients who are hospitalized for an acute stroke. Glucose monitoring on admission for all patients with acute stroke may detect patients without previous known diabetes or prediabetes diagnosis [7]. Our study demonstrated the importance of detection of prediabetes and newly diagnosed diabetes in acute stroke patients. Patients seen with lower average blood glucose levels during their hospitalization, have lower admission NIH Stroke Scores and lower discharge NIH Stroke Scores. Lower blood glucose levels during hospitalization also led to decreased length of stay, increase discharge to home, and decrease in hospital mortality.

Our study demonstrated the positive correlation between average blood glucose with the admission and discharge NIH stroke scale scores. Although prior studies have shown various outcomes with different ranges of inpatient glycemic control in patients with acute stroke, most studies indicated that hyperglycemia in acute stroke carries a poor prognostic indicator $[12,15,16]$. Hyperglycemia is thought to increase blood brain barrier penetration, increase issue acidosis, and cause free-radical related tissue injury, all leading to additional neuronal injury in an acute stroke $[12,16]$. Chronic hyperglycemia has been linked with several stroke risk factors including accelerated atherosclerosis, increased carotid intima media thickness (CIMT), cardiomyocyte dysfunction, atrial fibrillation (AF), and ischemic heart disease [17]. Other studies have shown that extremely tight glycemic control may not show benefit in outcomes of patients with acute stroke. A recent study of over 1100 adults with hyperglycemia in acute stroke, showed that there was no difference of favorable functional outcomes in patients whose blood glucose was between $80-130 \mathrm{mg} / \mathrm{dl}$, compared to 80 $180 \mathrm{mg} / \mathrm{dl}$ [13]. Patients with tighter glycemic control were more likely to experience hypoglycemia, which can lead to more neurological damage and complications.

Studies from the CDC have shown that the overall length of stay for patients with acute stroke has significantly decreased over the past 30 years, decreasing from 10.2 days in 1989 to 5.3 days in 2009 [1,8]. Prior studies have shown that certain factors may increase length of stay in patients hospitalized with acute stroke $[1,7,11,2]$. These factors include presence of ataxia, recurrent stroke, living alone, and absence of a caregiver at admission [3]. This study demonstrates the potential impact of glycemic control with length of stay and overall disposition of patients who are 
hospitalized with stroke. This study reveals that those patients with better glycemic control had significantly lower length of stay when compared to those patients with higher admission blood sugars [3, 8].

In this small sample sized study, the results are clearly consistent with other study. Patient with lower average blood glucose level during their hospitalization has a lower admission and discharge NIH Stroke Score. The lower average glucose also led to decreased length of stay and increased discharge to home rate. Interestingly, this study found that higher BMI $(>30)$ had higher discharge NIH stroke scale and lower home discharge rate. Even though this difference was not statistically significant, we would assume that with bigger sample size, this difference may be significant. Further studies needed to determine if strict blood sugar control is needed during acute and subacute phase of ischemic stroke.

With the information gathered in this study, we plan to develop and implement a hyperglycemia management protocol in our Comprehensive Stroke Center; with this new protocol, we will aim to educate the stroke nurses and supporting staff to work toward better patient outcomes in our patients admitted for acute stroke.

\section{Limitation}

This study is limited by smaller sample size. There is no standard hyperglycemia protocol was followed in this study and all hyperglycemic patients were treated with intravenous insulin. Multiple confounding variables including comorbidities were not considered when determining the outcome of the stroke patient. While calculating the average blood glucose level, only lowest value and highest value was considered.

\section{Conclusion}

Although this study limited by smaller patient groups, but it concludes that higher blood glucose level associated with higher NIH stroke scale at admission and during discharge and have increased length of stay which is consistent with other similar studies.

\section{Funding Sources}

This project was not supported by any grant or funding agencies.

\section{Conflict of Interest Statement}

The authors declare that they have no competing interest.

\section{References}

[1] Lau, L. H., et al., Prevalence of diabetes and its effects on stroke outcomes: A meta-analysis and literature review. Journal of diabetes investigation, 2019. 10 (3): p. 780-792.
[2] Benjamin, E. J., P. Muntner, and M. S. Bittencourt, Heart disease and stroke statistics-2019 update: a report from the American Heart Association. Circulation, 2019. 139 (10): p. e56-e528.

[3] Chen, R., B. Ovbiagele, and W. Feng, Diabetes and stroke: epidemiology, pathophysiology, pharmaceuticals and outcomes. The American journal of the medical sciences, 2016. 351 (4): p. 380-386.

[4] Gaillard, T. and E. Miller, Guidelines for Stroke Survivors with Diabetes Mellitus. Stroke, 2018. 49 (6): p. e215-e217.

[5] Semenkovich, C. F., We know more than we can tell about diabetes and vascular disease: The 2016 Edwin Bierman Award Lecture. Diabetes, 2017. 66 (7): p. 1735-1741.

[6] Pechlivani, N. and R. A. Ajjan, Thrombosis and vascular inflammation in diabetes: mechanisms and potential therapeutic targets. Frontiers in cardiovascular medicine, 2018. 5: p. 1 .

[7] Zhang, X., et al., Prevalence of abnormal glucose regulation according to different diagnostic criteria in ischemic stroke without a history of diabetes. BioMed research international, 2018 .

[8] Control, C. f. D. and Prevention, National diabetes statistics report, 2017. Atlanta, GA: Centers for Disease Control and Prevention, US Department of Health and Human Services, 2017. 20.

[9] Benjamin, E. J., et al., Heart disease and stroke statistics-2018 update: a report from the American Heart Association. Circulation, 2018. 137 (12): p. e67.

[10] Powers, W. J., et al., 2018 guidelines for the early management of patients with acute ischemic stroke: a guideline for healthcare professionals from the American Heart Association/American Stroke Association. Stroke, 2018. 49 (3): p. e46-e99.

[11] Emanuele, M. and N. Emanuele, Management of Diabetes in Stroke, in Primer on Cerebrovascular Diseases. 2017, Elsevier. p. $756-759$.

[12] Bruno, A., et al., Acute blood glucose level and outcome from ischemic stroke. Neurology, 1999. 52 (2): p. 280-280.

[13] Johnston, K. C., et al., Intensive vs standard treatment of hyperglycemia and functional outcome in patients with acute ischemic stroke: the SHINE randomized clinical trial. JAMA, 2019. 322 (4): p. 326-335.

[14] Spss, I., IBM SPSS statistics for Windows, version 20.0. New York: IBM Corp, 2011. 440.

[15] Weir, C. J., et al., Is hyperglycemia an independent predictor of poor outcome after acute stroke? Results of a long term follow up study. BMJ 1997. 314 (7090): p. 1303.

[16] Capes, S. E., et al., Stress hyperglycemia and prognosis of stroke in nondiabetic and diabetic patients: a systematic overview. Stroke, 2001. 32 (10): p. 2426-2432.

[17] Parsons, M. W., et al., Acute hyperglycemia adversely affects stroke outcome: a magnetic resonance imaging and spectroscopy study. Annals of Neurology: Official Journal of the American Neurological Association and the Child Neurology Society, 2002. 52 (1): p. 20-28. 